Arch. Tierz., Dummerstorf 46 (2003) 2, 113-126

Aus dem Institut für Tierzucht und Tierhaltung mit Tierklinik der Martin-Luther-Universität Halle-Wittenberg

HERMANN H. SWALVE und KERSTIN HÖVER

\title{
Untersuchungen an den Ergebnissen der Zuchtwertschätzung von Holstein Bullen und Kühen in Deutschland
}

\author{
Herrn Prof. Dr. agr. habil. Dr. h. c. mult. Georg Schönmuth zum 75. Geburtstag \\ gewidmet
}

\begin{abstract}
Summary
Title of the paper: Examinations using the results of breeding value estimation for Holstein sires and cows in Germany

Besides for selection purposes, estimated breeding values can be used to analyze genetic trends and structures in populations. Using data of 2,440,261 cows from four regions in Germany, genetic trends for all cows under test (registered and grade) were calculated for the period of birth years 1992 to 1999. For milk, fat, and protein yield annual trends on average were $111 \mathrm{~kg}, 3.2 \mathrm{~kg}$, and $3.3 \mathrm{~kg}$, respectively. An analysis of the use of proven sires revealed that a relatively large proportion of bulls get second crop daughters. Most of them, however, are used in a very limited way. The differential use of sires by breeders besides production is highly influenced by type proofs although in this respect clear differences exist between regions. An analysis comparing first proofs of young bulls with their later proofs based on additional second crop daughters for all German Holstein breeding organisations showed a high degree of agreement between the two proofs. However, this analysis was based on only 127 sires that were used more widely (plus $>500$ second crop daughters).
\end{abstract}

Key Words: Holstein population, genetic trend, proven sires

\section{Zusammenfassung}

Neben den Zwecken der Selektion können die Ergebnisse der Zuchtwertschätzung zur Analyse des genetischen Fortschritts und genetischer Strukturen in Populationen genutzt werden. An einem Datenmaterial von 2.440.061 Kühen aus vier Regionen in Deutschland wurde der genetische Fortschritt für die Population der Kühe unter Milchleistungsprüfung für die Periode der Geburtsjahre 1992 bis 1999 berechnet. Der jährliche genetische Fortschritt betrug im Mittel 111 kg Milch, 3,2 kg Fett und 3,3 kg Eiweiß. Eine Analyse der Einsatzhäufigkeit geprüfter Bullen zeigte, dass relativ viele Bullen in einen Wiedereinsatz gelangen. Die meisten dieser Bullen werden allerdings nur sehr beschränkt genutzt. Der differenzierte Gebrauch der Bullen durch die Züchter wird neben der Milchleistungsveranlagung insbesondere auch von den Zuchtwerten für Exterieur bestimmt, obschon in dieser Hinsicht auch klare Unterschiede zwischen Verbandsgebieten existieren. Eine Analyse des Vergleichs der Zuchtwerte aus Test- und Wiedereinsatz für Bullen aller deutschen Zuchtorganisationen zeigte eine hohe Übereinstimmung zwischen beiden Zuchtwerten. Allerdings konnten für diese Untersuchung lediglich 127 Bullen, die stärker eingesetzt worden waren (plus > 500 Töchter aus dem Wiedereinsatz), einbezogen werden.

Schlüsselwörter: Holstein Population, genetischer Fortschritt, geprüfte Bullen

\section{Einleitung}

Die Ergebnisse der Zuchtwertschätzung für Rinder, die für die Rasse Holstein von der Vereinigte Informationssysteme Tierhaltung (VIT) in Verden durchgeführt wird, werden üblicherweise in Fachzeitschriften hinsichtlich von Top-Listen für Bullen diskutiert. An den Ergebnissen der Zuchtwertschätzung lassen sich jedoch weitere Analysen durchführen, die Schlussfolgerungen hinsichtlich des Standes der Population zulassen. 
Von Interesse sind neben den Ergebnissen der Zuchtwertschätzung für Bullen hierbei insbesondere auch diejenigen für Kühe. In der vorliegenden Untersuchung sollen die Fragen des Zuchtfortschritts, der Ausnutzung der Bullen in Abhängigkeit von ihren Zuchtwerten, sowie die Zuchtwertentwicklung von Bullen von ihrem ersten Zuchtwert, basierend auf dem Testeinsatz, bis zum Zuchtwert aus dem Wiedereinsatz als geprüfte Bullen in einfacher Form dargestellt werden.

\section{Material und Methoden}

Das verwendete Datenmaterial umfasste Zuchtwerte von 3.043 Mill. Holstein-Kühen, die in den Jahren 1995 bis 2002 zu einer ersten, zweiten, oder dritten Laktation gekalbt hatten und aus den Zuchtgebieten Schleswig-Holstein, Niedersachsen (Gebiet Hannover der Zuchtorganisationen ZEH und RPN), Berlin-Brandenburg und MecklenburgVorpommern stammten. Das Material umfasste dabei alle Kühe unter Milchleistungsprüfung (MLP), also Herdbuch- und Nicht-Herdbuchkühe. Von jeder Kuh lag auch die Abstammung (Vater und Mutter) - soweit bekannt - vor. Ferner wurden hinsichtlich der Ergebnisse der Zuchtwertschätzung für Bullen die sog. ZWVITALL-Dateien der VIT aus unterschiedlichen Schätzterminen verwendet. Diese Dateien enthalten die Zuchtwerte aller KB-Bullen, die entweder Testbullen waren oder in den jüngsten zwei Kalbejahren Töchter hatten. Bullen mit weniger als 20 Töchtern und Bullen des Natursprungeinsatzes sind nicht enthalten.

\section{Genetischer Trend}

Zur Analyse des genetischen Trends in der Kuhpopulation wurden aus dem Gesamtmaterial alle schwarzbunten Holstein-Kühe der Geburtsjahre 1992 bis 1999 extrahiert. Damit verblieben 2.440.261 Kühe der vier Verbandsgebiete. Die Verteilung auf die Verbandsgebiete zeigt Tabelle 1. Da zwischen den Verbandsgebieten große Unterschiede hinsichtlich der Bekanntheit der Abstammung bestehen, wurde ein zweites Teilmaterial gebildet, welches nur Kühe enthielt, deren Abstammung (Vater und Mutter) bekannt war. Der Umfang dieses Datenmaterials ist ebenfalls in Tabelle 1 dargestellt.

Tabelle 1

Anzahl Kühe (MLP) der Geburtsjahre 1992 bis 1999 nach Verbandsgebiet (No. of cows on test (registered and grade) of birth years 1992 to 1999 by region)

\begin{tabular}{lll}
\hline Zuchtgebiet & Alle Kühe & $\begin{array}{l}\text { Kühe mit bekannter Abstammung } \\
\text { (Vater und Mutter) }\end{array}$ \\
\hline Schleswig-Holstein & 464.728 & 274.199 \\
Niedersachsen (Hannover) & 1.017 .176 & 919.374 \\
Berlin-Brandenburg & 495.653 & 430.854 \\
Mecklenburg-Vorpommern & 462.704 & 348.993 \\
\hline
\end{tabular}

Beziehungen zwischen Zuchtwert und Einsatzhäufigkeit der Bullen

Zur Untersuchung der Frage der möglichen Beziehungen zwischen Zuchtwert und Einsatzhäufigkeit der Bullen wurde ein Datenmaterial von Jungkühen der Geburtsjahrgänge 1998 und 1999 aus dem Gesamtmaterial ausgewählt. Vorbedingung für eine derartige Analyse ist natürlich das Vorhandensein einer väterlichen Abstammung, die Bekanntheit einer Mutternummer wurde hier nicht vorausgesetzt. Tabelle 2 zeigt das so definierte Datenmaterial. Aufgrund der o.a. Eigenschaften der ZWVITALL-Dateien - verwendet wurde die Datei des Schätzlaufes August 2002 - erstreckt sich die Ana- 
lyse lediglich auf den in den beiden rechten Spalten der Tabelle 2 angegebenen Datenumfang. Es zeigt sich, dass in allen Verbandsgebieten bedeutende Anzahlen von Bullen herausfallen, die jedoch, da es sich hierbei überwiegend um Natursprungbullen und erst im sehr kleinen Umfang eingesetzte Natursprungbullen handelt, nur für einen vergleichsweise kleinen Teil der Kühe als Väter verzeichnet sind.

Tabelle 2

Anzahl Kühe (MLP) der Geburtsjahre 1998/99 mit bekannten Vätern nach Verbandsgebiet (No. of cows on test (registered and grade) of birth years 1998/99 by region)

\begin{tabular}{lllll}
\hline Zuchtgebiet & $\begin{array}{l}\text { Gesamtmaterial } \\
\text { (Vater bekannt) }\end{array}$ & & \multicolumn{2}{l}{$\begin{array}{l}\text { Material in der Analyse } \\
\text { (Vater in KB-Bullendatei) }\end{array}$} \\
\cline { 2 - 5 } & Anzahl Kühe & Anzahl Väter & Anzahl Kühe & Anzahl Väter \\
\hline Schleswig-Holstein & 65.481 & 2.135 & 56.287 & 858 \\
Niedersachsen (Hannover) & 204.297 & 6.053 & 174.773 & 1.998 \\
Berlin-Brandenburg & 103.334 & 2.538 & 94.579 & 1.536 \\
Mecklenburg-Vorpommern & 86.819 & 2.985 & 71.459 & 1.452 \\
\hline
\end{tabular}

Beziehungen zwischen Zuchtwert aus Testeinsatz und Wiedereinsatz

Für diese Analyse wurden die ZWVITALL-Dateien verwendet. Ausgangspunkt waren die Ergebnisse der Zuchtwertschätzung August 1999. Zu diesem Zeitpunkt war der Testeinsatz des Geburtsjahrgangs 1993 in nahezu allen Verbänden abgeschlossen, d.h. die Ergebnisse der Zuchtwertschätzung lagen vor Der Jahrgang 1994 war noch unvollständig, der Jahrgang 1995 erst mit 13 Bullen vertreten. Als abgeschlossen wurden Zuchtwerte (Milch bzw. RZM) mit einer Sicherheit von > 70 \% betrachtet. Auch Bullen der Jahrgänge 1991 und 1992 wurden hinzugenommen, da in diesen der Testeinsatz zwar meist schon abgeschlossen war, jedoch noch keine Töchter aus einem Wiedereinsatz vorhanden waren.

Die Selektionskriterien waren:

Holstein-Schwarzbunt

Sicherheit RZM > 70 \%

Geburtsjahre 1991 bis 1995

Testeinsatz mit $<300$ Töchtern

Nur Töchter aus dem Testeinsatz im Zuchtwert

Nur Testbullen der 13 wichtigen Schwarzbunt-Stationen

Die so erhaltene Datei umfasste 2.672 Bullen. Diese Datei wurde dann mit der Datei der Zuchtwertschätzung Mai 2002 zusammengespielt. Die ZWVITALL-Datei Mai 2002 - und nicht die aktuelle Datei August 2002 - wurde verwendet, da die Formel für den Relativzuchtwert Milch (RZM) im August 2002 angepasst wurde und somit nicht mit der alten Formel vergleichbar gewesen wäre.

\section{Tabelle 3}

Verteilung der Bullen (Holstein, alle Verbände), die zum Zeitpunkt August 1999 noch keine Wiedereinsatztöchter hatten auf Geburtsjahrgänge (Distribution of bulls (Holstein, all organisations) which did not have second crop daughters in August 1999 by birth year)

\begin{tabular}{cc}
\hline Geburtsjahr & Anzahl \\
\hline 1991 & 586 \\
1992 & 667 \\
1993 & 769 \\
1994 & 637 \\
1995 & 13 \\
\hline Total & 2672 \\
\hline
\end{tabular}


Tabelle 3 zeigt die aus der ZWVITALL-Datei 08/99 selektierte Datei mit der Verteilung über Geburtsjahre. Der stärkste Jahrgang ist 1993, da der Testeinsatz gerade abgeschlossen war und noch keine Töchter aus dem Wiedereinsatz vorhanden waren. Ab 1994 sind die Jahrgänge noch unvollständig; vor 1993 sind sie einerseits deshalb kleiner als 1993, weil hier Bullen eliminiert wurden, die schon Töchter aus dem Wiedereinsatz hatten, und andererseits deshalb, weil nicht so viele Bullen getestet wurden wie 1993.

\section{Ergebnisse und Diskussion}

\section{Genetischer Trend}

Die Ergebnisse der Schätzung des genetischen Fortschritts (mittlere Kuhzuchtwerte je Geburtsjahrgang) je Jahr sind für die vier Verbandsgebiete in den Abbildungen 1 bis 10 dargestellt. Alle Abbildungen zeigen eine deutliche Differenzierung zwischen den Verbandsgebieten. Interessant ist insbesondere der Vergleich der beiden Verbandsgebiete der Neuen Bundesländer in Relation zur Entwicklung in den Alten Bundesländern. Die Gebiete Berlin-Brandenburg und Mecklenburg-Vorpommern starten im Betrachtungszeitraum mit dem Geburtsjahr 1992 in den Mengenmerkmalen Milchmenge (Abb. 1), Fettmenge (Abb. 2) und Eiweißmenge (Abb. 4) auf einem niedrigen Niveau, welches sich allerdings insbesondere ab dem Geburtsjahr 1996 dem Niveau der Verbandsgebiete Niedersachsen-Hannover und Schleswig-Holstein annähert; diese werden von den Verbandsgebieten der Neuen Bundesländer im jüngsten Geburtsjahrgang 1999 vollständig eingeholt. Die Entwicklung des genetischen Trends für die Inhaltsstoffe (Abb. 3 und 5) ist in allen Verbandsgebieten rückläufig, wobei die Verbände Berlin-Brandenburg und Mecklenburg-Vorpommern ihre anfänglich deutliche Überlegenheit hinsichtlich des Eiweißgehaltes vollständig verlieren und lediglich noch Vorteile im Fettgehalt aufweisen. Diese Entwicklung ist eine Funktion des Zuchtzieles, welches mit dem im Jahr 2002 abgelösten alten RZM einen deutlichen Schwerpunkt auf die Eiweißmenge legte.

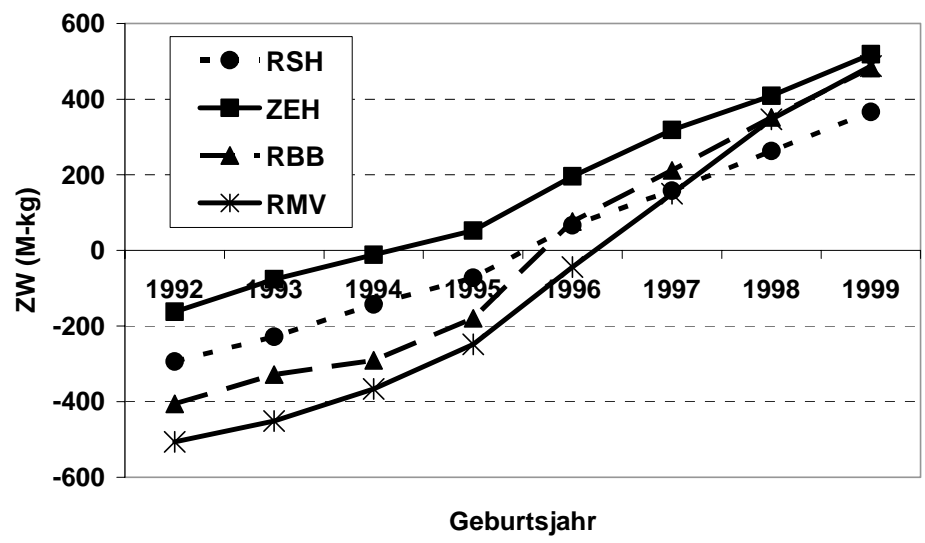

Abb. 1: Entwicklung der Zuchtwerte (alle MLP-Kühe) nach Verbandsgebieten: Milchmenge (kg) (RSH = Schleswig-Holstein, ZEH = Niedersachsen-Hannover, RBB = Berlin-Brandenburg, RMV = Mecklenburg-Vorpommern) (Development of breeding values (all cows on test) by region: Milk yield (kg))

Der Vergleich der Entwicklungen im RZM (Abb. 7) zeigt eine geringere Differenzierung zwischen den Verbandsgebieten. Mit dem Geburtsjahr 1996 erreichen die Verbände der Neune Bundesländer das Niveau derjenigen der Alten Bundesländer und übertreffen diese im jüngsten Geburtsjahr leicht. Der genetische Trend je Jahr ist graphisch in Abbildung 8 für das Merkmal RZM dargestellt. Auffällig ist insbesondere 
die sehr hohe Fortschrittsrate des Verbandsgebietes Mecklenburg-Vorpommern mit ca. 3,5 RZM-Punkten während das Verbandsgebiet Berlin-Brandenburg starke Schwankungen aufweist. Die Verbandsgebiete Niedersachsen-Hannover und Schleswig-Holstein weisen Steigerungsraten von ca. knapp 2 RZM-Punkten je Jahr auf.

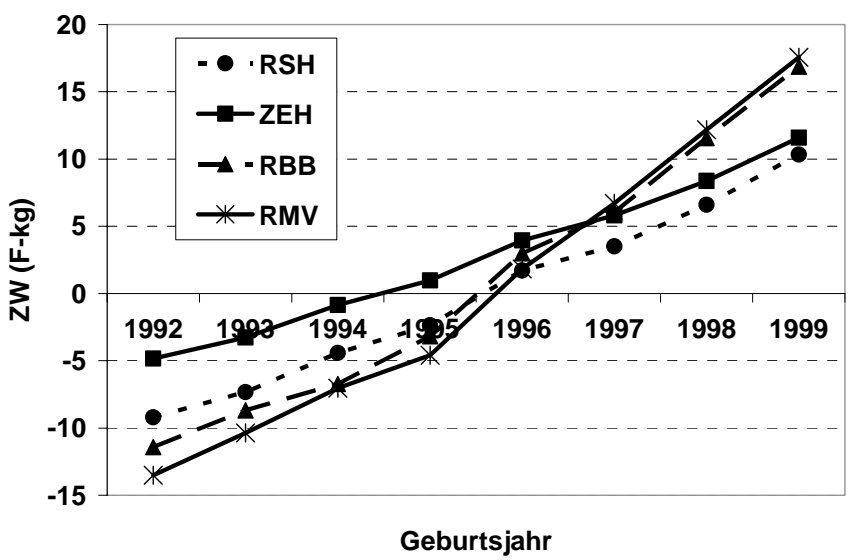

Abb. 2: Entwicklung der Zuchtwerte (alle MLP-Kühe) nach Verbandsgebieten: Fettmenge (kg) (RSH = Schleswig-Holstein, ZEH = Niedersachsen-Hannover, RBB = Berlin-Brandenburg, RMV = Mecklenburg-Vorpommern) (Development of breeding values (all cows on test) by region: Fat yield (kg))

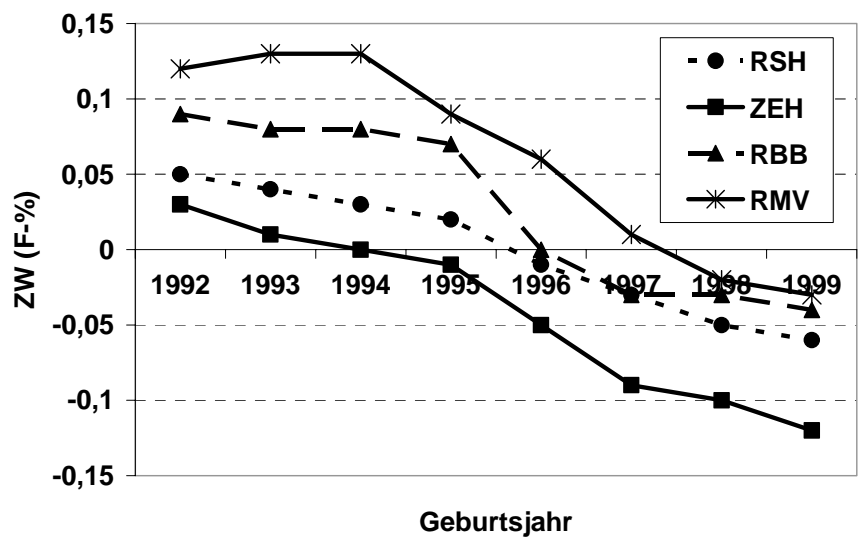

Abb. 3: Entwicklung der Zuchtwerte (alle MLP-Kühe) nach Verbandsgebieten: Fettgehalt (\%) (RSH = Schleswig-Holstein, ZEH $=$ Niedersachsen-Hannover, RBB $=$ Berlin-Brandenburg, RMV $=$ MecklenburgVorpommern) (Development of breeding values (all cows on test) by region: Fat content (\%))

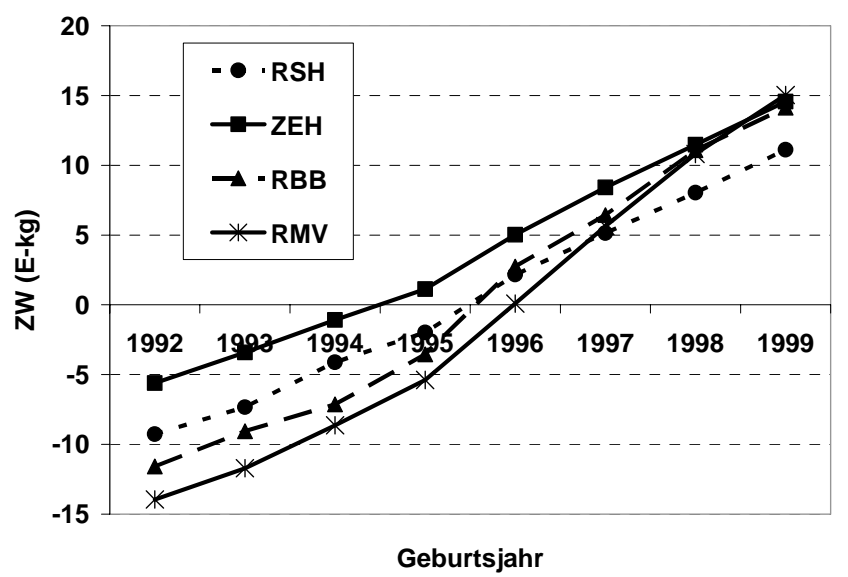

Abb. 4: Entwicklung der Zuchtwerte (alle MLP-Kühe) nach Verbandsgebieten: Eiweißmenge (kg) (RSH = Schleswig-Holstein, ZEH = Niedersachsen-Hannover, RBB = Berlin-Brandenburg, RMV = Mecklenburg-Vorpommern) (Development of breeding values (all cows on test) by region: Protein yield (kg)) 


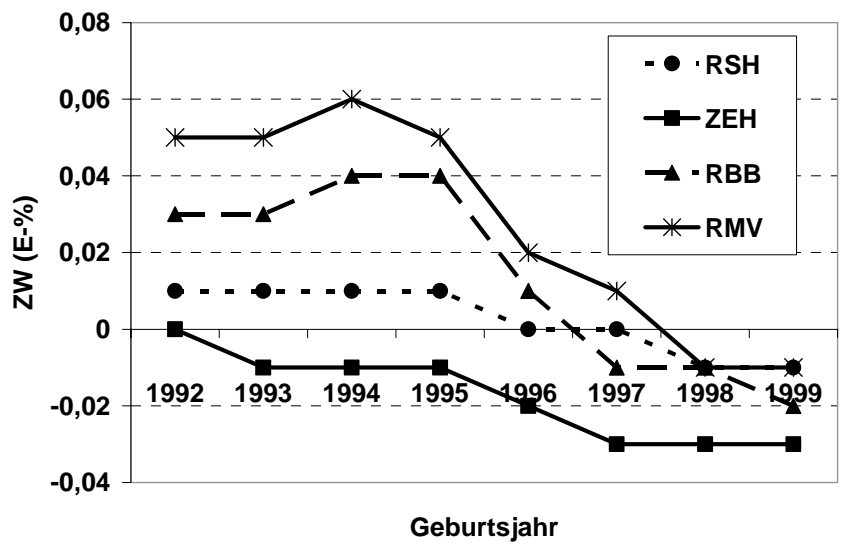

Abb. 5: Entwicklung der Zuchtwerte (alle MLP-Kühe) nach Verbandsgebieten: Eiweißgehalt (\%) (RSH = Schleswig-Holstein, ZEH = Niedersachsen-Hannover, RBB = Berlin-Brandenburg, RMV = Mecklenburg-Vorpommern) (Development of breeding values (all cows on test) by region: Protein content (\%))

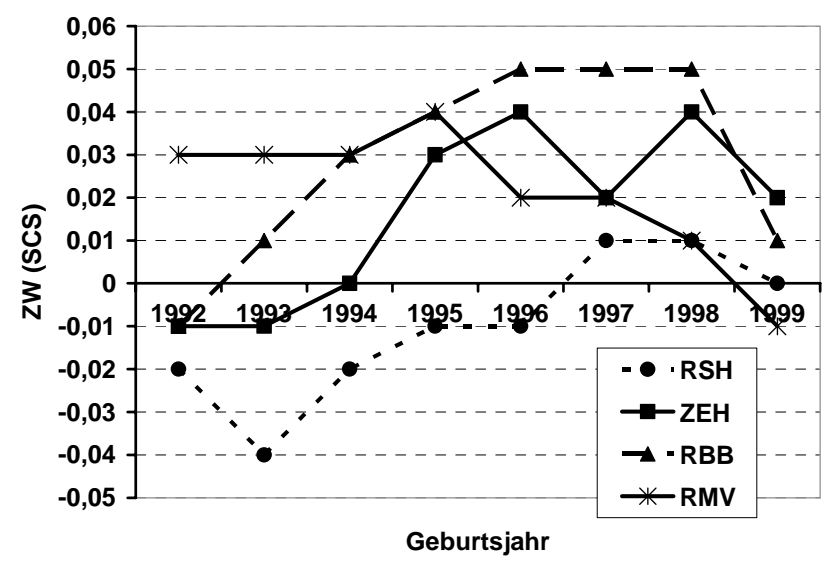

Abb. 6: Entwicklung der Zuchtwerte (alle MLP-Kühe) nach Verbandsgebieten: Somatic Cell Score (RSH = Schleswig-Holstein, ZEH = Niedersachsen-Hannover, RBB = Berlin-Brandenburg, RMV = Mecklenburg-Vorpommern) (Development of breeding values (all cows on test) by region: Somatic Cell Score)

In Abbildung 6 ist der Vollständigkeit halber auch der genetische Trend im Merkmal Zellzahl (SCS = Somatic Cell Score) dargestellt. Zur Interpretation der Graphik sind allerdings zwei Punkte bedeutsam: Erstens zeigt die Graphik die mittleren Zuchtwerte für den SCS auf der logarithmierten Originalskala, d.h. höhere Werte sind - wie auch international in vielen Ländern, aber sonst in Deutschland nicht üblich - züchterisch als negativ zu interpretieren. Hiernach könnte man somit einen deutlich negativen Trend für alle Verbandsgebiete außer Mecklenburg-Vorpommern ableiten. Dies relativiert sich jedoch, da zweitens hinzugefügt werden muss, dass die Standardabweichung der hier dargestellten Werte ca. 0,33 beträgt. Mithin liegen die dargestellten Veränderungen auf einem extrem niedrigen Niveau (ca. 1/10 der Standardabweichung) und sind somit kaum als echte Veränderungen zu bezeichnen. Ein nennenswerter züchterischer Fortschritt im Merkmal Zellzahl wird in keinem Verbandsgebiet erzielt - allerdings auch keine Verschlechterung. Im jüngsten Geburtsjahr verzeichnen alle Verbandsgebiete scheinbar eine positive Entwicklung. Die Ursache ist jedoch vermutlich ein Artefakt, da bei einem Merkmal mit niedriger Heritabilität wie der Zellzahl die Zuchtwerte noch junger Kühe aufgrund der niedrigeren Sicherheit besonders stark zum Mittelwert hin regressiert werden. 


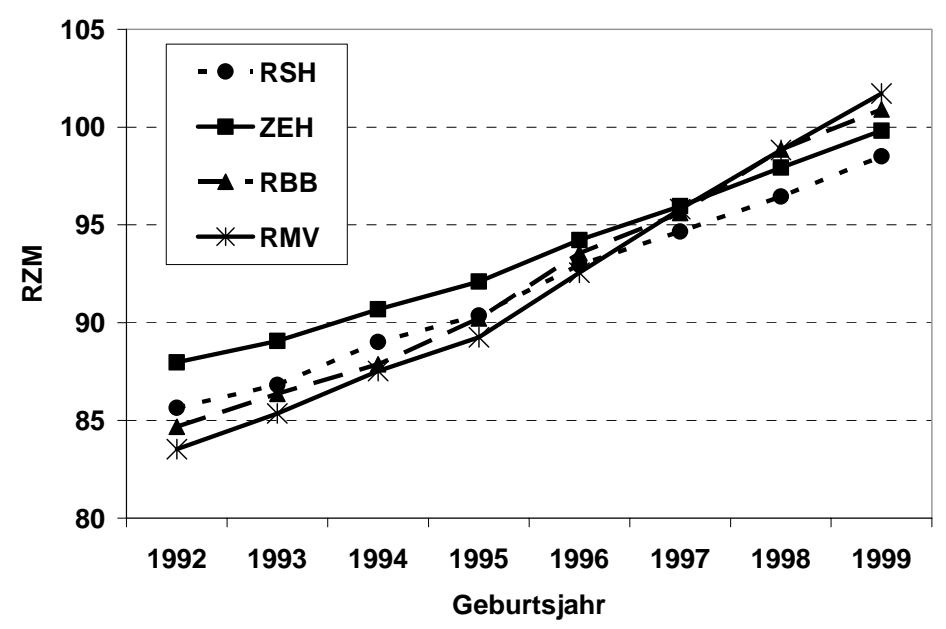

Abb. 7: Entwicklung der Zuchtwerte (alle MLP-Kühe) nach Verbandsgebieten: Relativzuchtwert Milch (RSH = Schleswig-Holstein, ZEH = Niedersachsen-Hannover, RBB = Berlin-Brandenburg, RMV = Mecklenburg-Vorpommern) (Development of breeding values (all cows on test) by region: Relative breeding value (production))

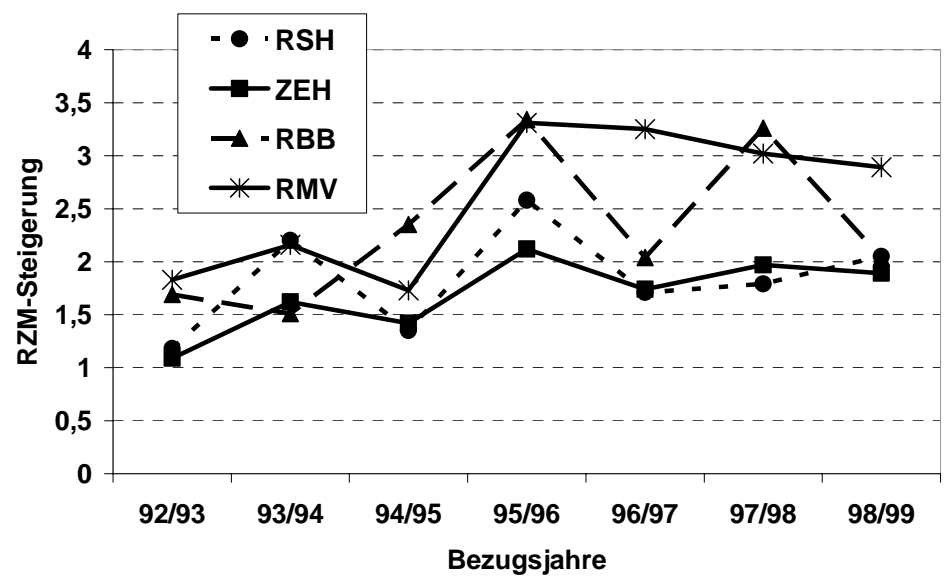

Abb. 8: Mittlerer Zuchtfortschritt je Jahr (alle MLP-Kühe) nach Verbandsgebieten: Relativzuchtwert Milch (RSH = Schleswig-Holstein, ZEH = Niedersachsen-Hannover, RBB = Berlin-Brandenburg, RMV = Mecklenburg-Vorpommern) (Annual genetic trend (all cows on test) by region: Relative breeding value (production))

Wie aus Tabelle 1 ersichtlich war, bestehen deutliche Unterschiede zwischen den Verbandsgebieten hinsichtlich der Bekanntheit der Abstammung. Im Verbandsgebiet Schleswig-Holstein liegen im Betrachtungszeitraum und unter den Bedingungen der Auswahl des Datenmaterials lediglich für 59 \% aller MLP-Kühe beide Eltern als bekannt vor. In allen anderen betrachteten Verbandsgebieten liegt dieser Wert deutlich höher, wobei Mecklenburg-Vorpommern eine Zwischenstellung einnimmt. Aus diesem Grund erschien eine zweite Analyse des genetischen Fortschritts an einem Teilmaterial angebracht, welches lediglich die Kühe umfasste, deren Abstammung bekannt war. Für die Entwicklung des RZM und den Fortschritt im RZM je Jahr sind die Ergebnisse in den Abbildungen 9 und 10 dargestellt. Tatsächlich zeigt sich durch die Einschränkung des Datenmaterials insbesondere für das Verbandsgebiet SchleswigHolstein ein anderes Bild. Schleswig-Holstein behauptet ab dem Geburtsjahr 1994 den Spitzenplatz. Hinsichtlich des genetischen Fortschritts je Jahr im Vergleich der Abbildungen 8 und 10 ergeben sich jedoch keine neuen Erkenntnisse. 


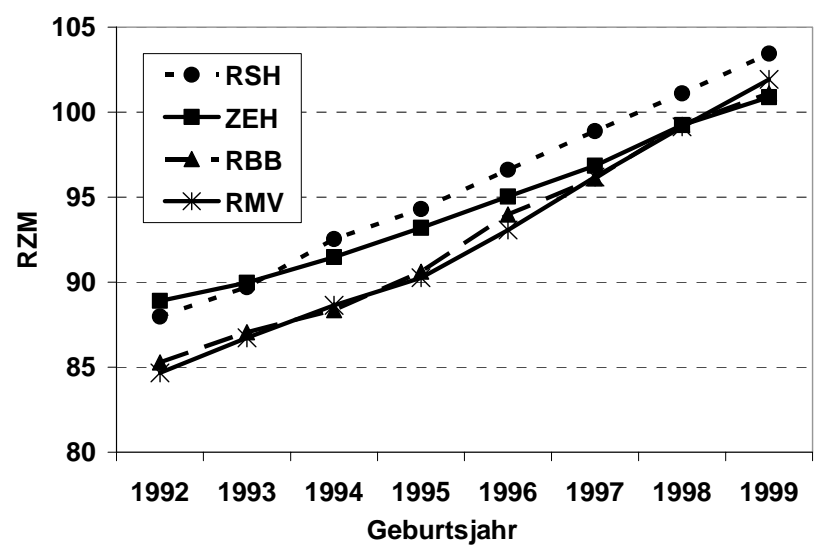

Abb. 9: Entwicklung der Zuchtwerte (alle MLP-Kühe mit bekannter Abstammung) nach Verbandsgebieten: Relativzuchtwert Milch $(\mathrm{RSH}=$ Schleswig-Holstein, $\mathrm{ZEH}=$ Niedersachsen-Hannover, $\mathrm{RBB}=$ BerlinBrandenburg, RMV = Mecklenburg-Vorpommern) (Development of breeding values (all cows on test with identified sire) by region: Relative breeding value (production))

Über die vier Verbandsgebiete hinweg ergibt sich in der MLP-Kuhpopulation (erste Analyse ohne Ausschluss von Kühen ohne bekannte Abstammung) ein genetischer Trend von 111 kg Milch, 3,2 kg Fett und 3,3 kg Eiweiß. In der klassischen Arbeit von VAN VLECK (1981) wird 100 kg Milch als in einem herkömmlichen Besamungszuchtprogramm realistischer Wert genannt. Bei einem Vergleich ist allerdings zu berücksichtigen, dass dieser Wert aufgrund der damaligen Schätzwerte für die genetische Varianz ermittelt wurde, welche sich durch die Leistungsentwicklung durch Skaleneffekte erhöht hat. Weiter ist zu bedenken, dass die heutigen Zuchtprogramme im Sinne der damals fast visionär anmutenden Vorschläge von NICHOLAS und SMITH (1983) zur starken Einbeziehung biotechnologischer Maßnahmen in Zuchtprogrammen mittlerweile viele dieser Vorschläge aufgegriffen haben und ein höherer Zuchtfortschritt mithin zu erwarten ist.

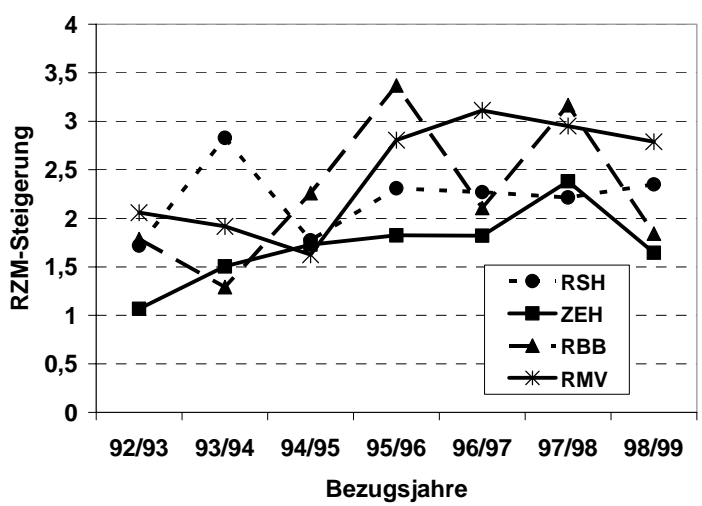

Abb. 10: Mittlerer Zuchtfortschritt je Jahr (alle MLP-Kühe mit bekannter Abstammung) nach Verbandsgebieten: Relativzuchtwert Milch $(\mathrm{RSH}=$ Schleswig-Holstein, $\mathrm{ZEH}=$ Niedersachsen-Hannover, $\mathrm{RBB}=$ BerlinBrandenburg, RMV = Mecklenburg-Vorpommern) (Annual genetic trend (all cows on test with identified sire) by region: Relative breeding value (production))

Schätzungen des genetischen Trends in Kuhpopulationen werden nur spärlich veröffentlicht und sind häufig mit dem Problem der Ziehung einer gewissen Stichprobe behaftet. Dies zeigt die Offenlegung des genetischen Trends für die Holstein-Populationen der USA und Kanada durch die verantwortlichen Stellen AIPL (Animal Improvement Programs Laboratory, USA) und CDN (Canadian Dairy Network) deren WebSeiten (AIPL, 2003; CDN, 2003) für einen Vergleich herangezogen wurden. Für die 
USA errechnen sich für einen dem dieser Studie identischen Betrachtungszeitraum 109 kg Milch, 3,3 kg Fett und 3,4 kg Eiweiß, also fast exakt die in der vorliegenden Studie gefundenen Werte. Zu berücksichtigen ist aber, dass das AIPL für die einzeln aufgeführten Geburtsjahre jeweils Stichprobengrößen von zwischen 600.000 und 700.000 Kühen angibt, also Zahlen, die keineswegs dem gesamten Kuhbestand der USA unter MLP entsprechen. Noch drastischer sind die Angaben des CDN. Hier wird für den Zeitraum 1992 bis 1997 ein Fortschritt von 172 kg Milch, 4,7 kg Fett und 5,4 kg Eiweiß angegeben, allerdings nur für eine Stichprobe von ca. 100.000 (Herdbuch-?) Kühen. Es kann mithin zumindest für den Vergleich mit den USA davon ausgegangen werden, dass der genetische Fortschritt je Jahr in den für die deutsche Population wohl repräsentativen vier Verbandsgebieten demjenigen in Nordamerika entspricht. Zu berücksichtigen ist aber der im Vergleich Deutschland - USA (nicht Deutschland - Kanada) noch immer vorhandene absolute Niveauunterschied. Hierauf weist die Studie von KÖNIG und SWALVE (2000) eindeutig hin. König und Swalve ermittelten für den Vergleich Deutschland - Niederlande sogar sich immer mehr vergrößernde Differenzen zugunsten der Niederlande in den Merkmalen der Milchleistung bei umgekehrten Verhältnissen in Bezug auf das Exterieur. Ursache hierfür ist die starke Gewichtung des Exterieurs bei deutschen Zuchtorganisationen und insbesondere auch in der Züchterschaft.

\section{Beziehungen zwischen Zuchtwert und Einsatzhäufigkeit der Bullen}

Zur Selektion von Bullen- und Kuhvätern sowie von Bullenmüttern werden in den Zuchtorganisationen vornehmlich die geschätzten Zuchtwerte und innerhalb dieser die auf die Basis der aktuellen Testbullenjahrgänge bezogenen Relativzuchtwerte RZM (Milch), RZE (Exterieur) und insbesondere RZG (Gesamt) genutzt. Für die Fortentwicklung einer Kuhpopulation ist jedoch mitentscheidend, wie stark überlegende Bullen auch ausgenutzt werden. Für die aktuellen Geburtsjahrgänge 1998 und 1999 aus den betrachteten vier Verbandsgebieten ist dies in den Abbildungen 11 bis 14 dargestellt. Zur Interpretation ist zu berücksichtigen, dass

- die Analyse durch die Beschränkung auf die aktuellen Geburtsjahrgänge eine Momentaufnahme ist, die auch durch einzelne im Zuchtwert herausragende Bullen bestimmt werden kann,

- das in Tabelle 2 beschriebene Datenmaterial auch Testbullen enthielt, deren Beitrag jedoch leicht gesondert mit einer Beschränkung auf die beiden kleinsten Töchterzahlklassen betrachtet werden kann.

In keinem Verbandsgebiet zeigen sich nennenswerte Beziehungen zwischen Einsatzhäufigkeit und RZM bzw. RZE der Bullen innerhalb der selektierten Stichprobe der überhaupt im Angebot befindlichen Bullen. Recht viele Bullen gehen überhaupt in einen Wiedereinsatz, meist jedoch in einen sehr beschränkten. Aus theoretischer Sicht wird üblicherweise unterstellt, dass erst Bullen, die im Zuchtwert über eine gewisse Marktschwelle hinausreichen, überhaupt vermarktet werden können (DEKKERS et. al., 1996). In der Population ist der Effekt der stark eingesetzten Bullen insgesamt natürlich dominierend, wobei angemerkt sei, dass die nach oben offene Klasse der Bullen mit mehr als 5.000 Töchtern häufig Bullen mit bis zu ca. 20.000 Töchtern enthielt. So wird auch verständlich, weshalb trotz der fehlenden Beziehung zwischen Zuchtwerten der Kuhväter und Einsatzhäufigkeit der o. a. Zuchtfortschritt erzielt wird. 


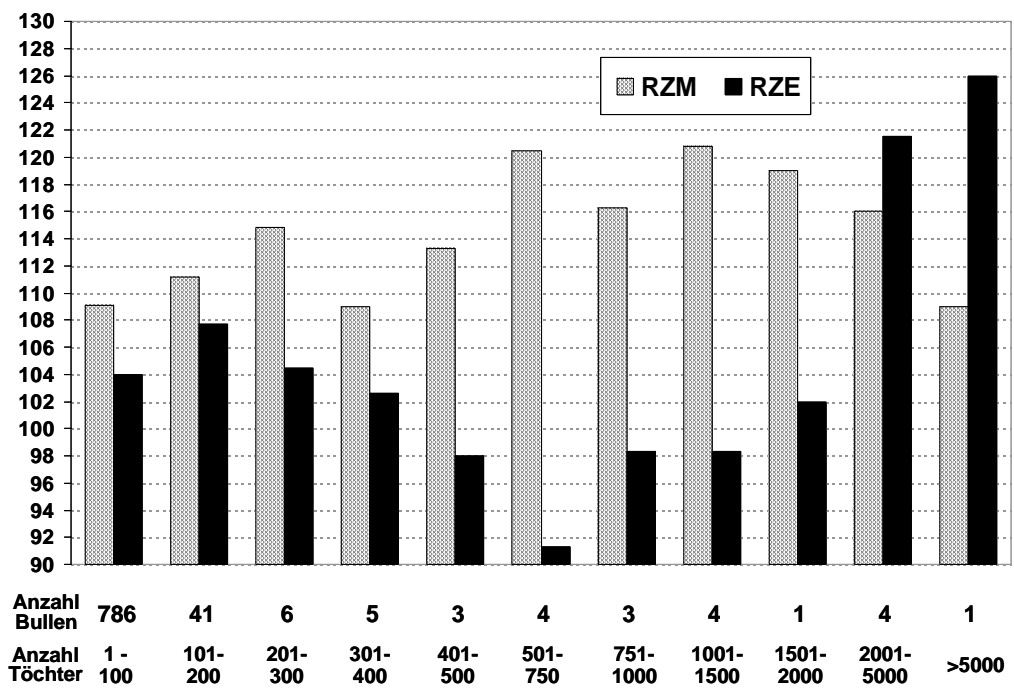

Abb. 11: Mittelwerte für RZM (Milch) und RZE (Exterieur) nach Töchterzahlklassen für 858 schwarzbunte Holstein-Bullen, die Väter von Kühen der Geburtsjahre 1998 und 1999 im Gebiet Schleswig-Holstein waren (Means of relative breeding values for milk (RZM) and type (RZE) by class of no. of daughters of 858 blackand-white Holstein bulls which were sires of cows of birth years 1998 and 1999 in the region Schleswig-Holstein)

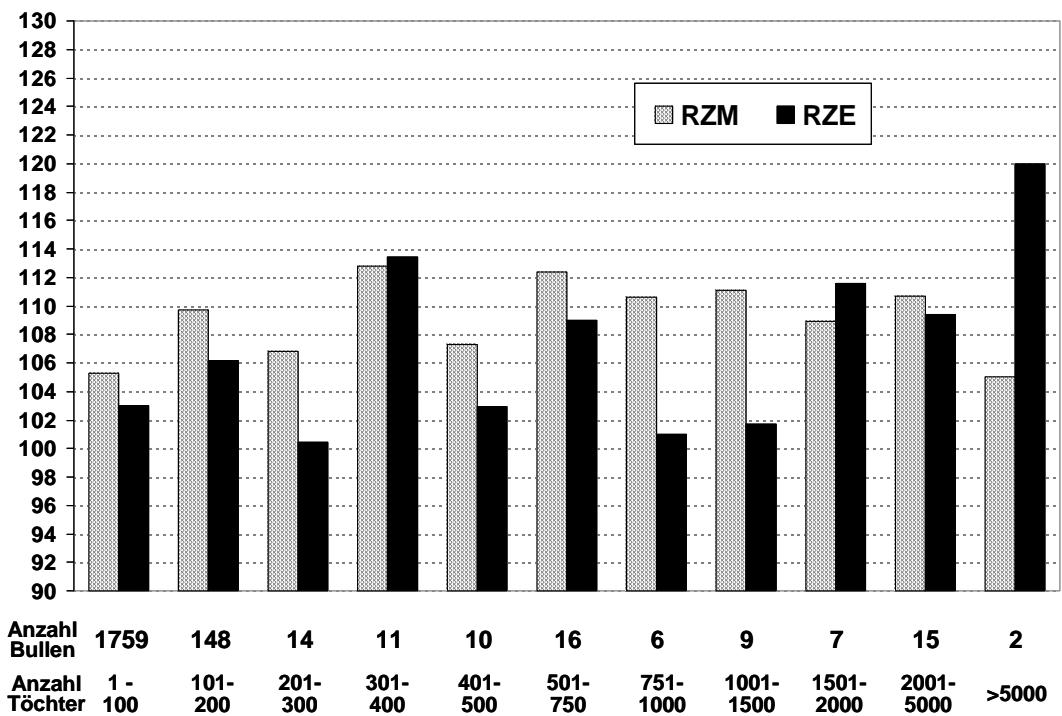

Abb. 12: Mittelwerte für RZM (Milch) und RZE (Exterieur) nach Töchterzahlklassen für 1996 schwarzbunte Holstein-Bullen, die Väter von Kühen der Geburtsjahre 1998 und 1999 im Gebiet Niedersachsen-Hannover waren (Means of relative breeding values for milk (RZM) and type (RZE) by class of no. of daughters of 1996 black-and-white Holstein bulls which were sires of cows of birth years 1998 and 1999 in the region Niedersachsen-Hannover)

Insgesamt liegen die Zuchtwerte der Bullen im Wiedereinsatz auf einem befriedigenden Niveau mit Abstufungen zwischen den Zuchtgebieten. Dies zeigt das Verbandsgebiet Niedersachsen-Hannover mit dem vergleichsweise niedrigsten Niveau im RZM gegenüber den drei anderen Gebieten und die starke Ausnutzung eines Top-Bullen (RZM 127, RZE 90; 7.505 Töchter) in Berlin-Brandenburg. ADLER (1999) wies bereits darauf hin, dass in Berlin-Brandenburg die Top-Bullen besonders stark ausgenutzt würden.

Die Abbildungen 11 bis 14 zeigen aber auch, dass hinsichtlich der Ausnutzung der Top-Bullen noch Reserven bestehen und dass bei den Landwirten häufig das Exterieur einen überproportional hohen Stellenwert einnimmt. SCHÖNMUTH (1999) erläuterte, 
dass die Geschichte der Schwarzbuntzucht in Deutschland einen ständigen Kampf zwischen einer Überbetonung des Exterieurs und einer angemessenen Berücksichtigung funktionaler Exterieurmerkmale beinhaltet hat. Diese Auseinandersetzung scheint nicht völlig der Geschichte anzugehören.

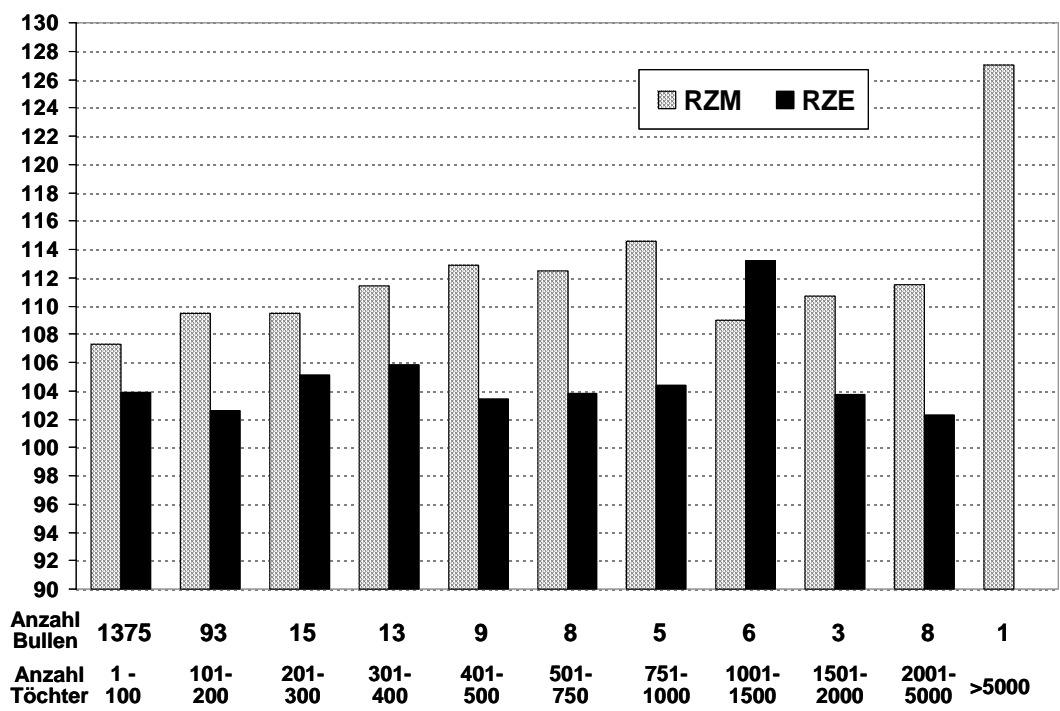

Abb. 13: Mittelwerte für RZM (Milch) und RZE (Exterieur) nach Töchterzahlklassen für 1536 schwarzbunte Holstein-Bullen, die Väter von Kühen der Geburtsjahre 1998 und 1999 im Gebiet Berlin-Brandenburg waren (Means of relative breeding values for milk (RZM) and type (RZE) by class of no. of daughters of 1536 blackand-white Holstein bulls which were sires of cows of birth years 1998 and 1999 in the region Berlin-Brandenburg)

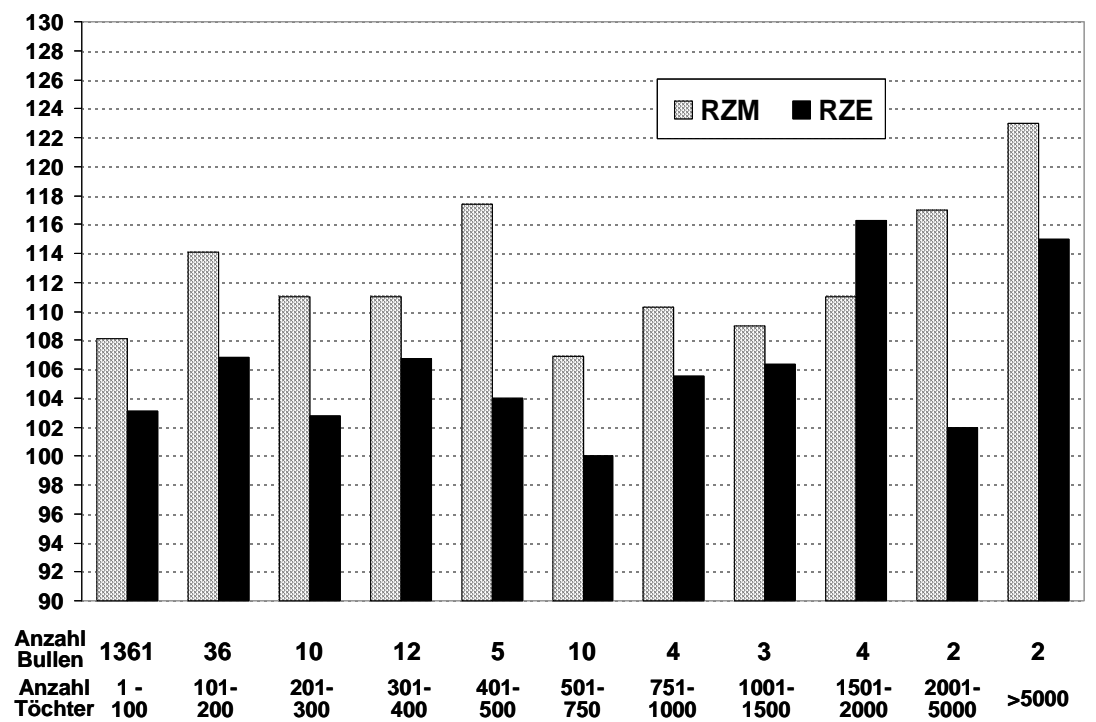

Abb. 14: Mittelwerte für RZM (Milch) und RZE (Exterieur) nach Töchterzahlklassen für 1449 schwarzbunte Holstein-Bullen, die Väter von Kühen der Geburtsjahre 1998 und 1999 im Gebiet Mecklenburg-Vorpommern waren (Means of relative breeding values for milk (RZM) and type (RZE) by class of no. of daughters of 1536 black-and-white Holstein bulls which were sires of cows of birth years 1998 and 1999 in the region Mecklenburg-Vorpommern)

Die insgesamt vergleichsweise hohen Zahlen der überhaupt verwendeten Bullen in der als exemplarisch für die deutsche Holstein-Population anzusehenden Stichprobe der vier Verbandsgebiete führt zu einer Besonderheit der deutschen Population. Nach Ergebnissen von SIMIANER und KÖNIG (2003) an hessischen Kühen liegt der mittlere 
Inzuchtgrad der Kühe derzeit lediglich bei 1 bis 1,5 \% und damit weit unter dem in anderen wichtigen Holstein-Ländern (z.B. USA, Kanada) ermittelten Inzuchtgrad von 4 bis $7 \%$. Das Verhalten der deutschen Milchviehhalter unterstützt damit die Bemühungen um eine Nachhaltigkeit in der Zucht im Sinne der Erhaltung einer genetischen Vielfalt.

\section{Beziehungen zwischen Zuchtwert aus Testeinsatz und Wiedereinsatz}

Die Verteilung auf die Verbände und die Zahl der je Verband in der ZWS 05/02 wiedergefundenen Bullen (wiedergefunden mit Wiedereinsatztöchtern) zeigt Tabelle 4. Es ist offenbar, dass die jüngeren Jahrgänge 1994 und 1995 als unvollständig betrachtet werden müssen, da diese Bullen eben noch keine Töchter aus dem Wiedereinsatz gehabt haben können. Ebenso gilt dies partiell auch für noch ältere Bullen, für die der Wiedereinsatz noch nicht abgeschlossen war. Auch diese Analyse bleibt somit eine Momentaufnahme.

Tabelle 4

Verteilung der Bullen (Holstein, alle Verbände), die zum Zeitpunkt August 1999 noch keine Wiedereinsatztöchter hatten, aber zum Zeitpunkt Mai 2002 Wiedereinsatztöchter aufwiesen auf Zuchtorganisationen (Distribution of bulls (Holstein, all organisations) which did not have second crop daughters in August 1999 but had second crop daughters in May 2002 by breeding organisation)

\begin{tabular}{llccc}
\hline Region & Station & Alle Testbullen & $\begin{array}{c}\text { Alle Bullen mit } \\
\text { Wiedereinsatz }\end{array}$ & $\begin{array}{c}\text { Mit Wiedereinsatz } \\
\text { plus }>500 \text { Töchter }\end{array}$ \\
\hline Baden-Württemberg & RBW & 37 & 12 & 3 \\
Thüringen & LTR & 135 & 13 & 7 \\
Osnabrück & OHG & 97 & 14 & 7 \\
Brandenburg & RBB & 221 & 37 & 11 \\
Mecklenburg-Vorpommern & RMV & 180 & 50 & 14 \\
Niedersachsen (Hannover) & RPN & 550 & 66 & 19 \\
Sachsen-Anhalt & RSA & 165 & 42 & 9 \\
Schleswig-Holstein & RSH & 232 & 33 & 9 \\
Westfalen & RUW & 367 & 40 & 12 \\
Sachsen & SRV & 174 & 54 & 10 \\
Ostfriesland & VOST & 160 & 17 & 9 \\
Weser-Ems & WEU & 227 & 16 & 8 \\
Hessen & ZBH & 127 & 15 & 9 \\
\hline Total & & 2672 & 409 & 127 \\
\hline
\end{tabular}

Wie schon im vorangegangenen Abschnitt deutlich wurde, gehen recht viele Bullen $(n=409)$ überhaupt in irgendeinen Wiedereinsatz, meist jedoch in einen sehr beschränkten. Deshalb zeigt die rechte Spalte der Tabelle 4 die Zahl der Bullen mit einem Wiedereinsatz, bei dem mindestens 500 Töchter hinzukamen.

Für den Vergleich der Zuchtwerte aus der Zuchtwertschätzung Mai 2002 mit derjenigen aus August 1999 ist zu berücksichtigen, dass der Vergleich der Zuchtwerte um die Basisanpassungen August 2000 und August 2001 korrigiert werden muss. Diese betrugen -1,3 (08/00) und -2,4 (08/01) RZM-Punkte und die erwartete Differenz beträgt mithin -3,7 Punkte.

Tabelle 5 zeigt den Vergleich (korrigiert um die Basisanpassung von -3,7 Punkten) für Bullen, die man als „echte“ Wiedereinsatzbullen betrachten könnte (plus > 500 Töchter aus dem Wiedereinsatz). Allerdings liegt auch in diesem Fall überwiegend ein regional begrenzter Wiedereinsatz vor. Die Ergebnisse des Vergleichs streuen um den nach der Korrektur erwarteten Wert von Null. Größere negative Abweichungen findet man für die Verbände Baden-Württemberg, sowie Hessen und Thüringen. Eine weitere 
Ausnahme ist der VOSt (Ostfriesland), hier ergibt sich sogar eine deutlich positive Differenz, die allerdings fast ausschließlich auf die stark positive Zuchtwertentwicklung eines einzigen Bullen zurückzuführen ist. Unter Herauslassung dieses Bullen beträgt die Differenz für Ostfriesland +2,4 Punkte.

\section{Tabelle 5}

Mittlere Differenz des Vergleichs RZM02 - RZM99 für 127 Bullen mit Wiedereinsatz (plus > 500 Töchter) nach Organisationen; korrigiert um Basisanpassungen. (Average difference of comparison RZM02 -RZM99 for 127 bulls with second crop daughters (plus $>500$ daughters) by organisation (RZM = relative breeding value for production); corrected for base shifts)

\begin{tabular}{lllll}
\hline Region & Station & $\mathrm{n}$ & Mittelwert & Standardabweichung \\
\hline Baden-Württemberg & RBW & 3 & $-3,30$ & 5,20 \\
Thüringen & LTR & 7 & $-3,30$ & 3,00 \\
Osnabrück & OHG & 7 & $+0,27$ & 5,03 \\
Brandenburg & RBB & 11 & $+0,15$ & 5,92 \\
Mecklenburg-Vorpommern & RMV & 14 & $-0,87$ & 6,96 \\
Niedersachsen (Hannover) & RPN & 19 & $+1,54$ & 4,96 \\
Sachsen-Anhalt & RSA & 9 & $-1,74$ & 4,39 \\
Schleswig-Holstein & RSH & 9 & $+2,14$ & 5,83 \\
Westfalen/Rheinland/Rheinland-Pfalz & RUW & 12 & $-2,47$ & 2,69 \\
Sachsen & SRV & 10 & $+0,10$ & 4,81 \\
Ostfriesland & VOST & 9 & $+3,81$ & 5,95 \\
Weser-Ems (Oldenburg/Emsland) & WEU & 8 & $+3,20$ & 4,78 \\
Hessen & ZBH & 9 & $-3,97$ & 4,50 \\
\hline
\end{tabular}

Das Beispiel Ostfriesland macht deutlich, dass die vorgelegte Analyse zur Entwicklung der Zuchtwerte aus dem Wiedereinsatz im Vergleich zum Testeinsatz mit dem Problem behaftet ist, dass schon einzelne Bullen in dieser sehr kleinen Stichprobe sehr große Schwankungen herbeiführen können. Die Ergebnisse sollten deshalb mit aller gebotenen Vorsicht interpretiert werden. Der grundsätzliche Ansatz der Analyse war, dass Bullen mit herausragenden Zuchtwerten aus dem Testeinsatz auch in einen starken überregionalen Wiedereinsatz gehen. Nach Sichtung des Datenmaterials muss aber gerade diese Annahme als nur sehr eingeschränkt gültig bezeichnet werden. Auch die Untergrenze von mindestens plus 500 Töchtern kann eigentlich noch kaum im Sinne eines starken Wiedereinsatzes interpretiert werden.

Unter den gegebenen Einschränkungen bei der Interpretation der vorliegenden Ergebnisse lassen sich folgende Tendenzen erkennen: Die häufig von Kritikern der Zuchtwertschätzung vorgebrachte Annahme, dass gerade Bullen aus sehr erfolgreichen Verbänden nach einem späteren Wiedereinsatz „abstürzen“, lässt sich aufgrund der vorliegenden Ergebnisse nicht erhärten, wie die Beispiele Sachsen, Osnabrück und Mecklenburg-Vorpommern zeigen. In wenigen Zuchtgebieten (Baden-Württemberg, Thüringen, Hessen und Rinder-Union West) sind jedoch Tendenzen zum „Abstürzen“ nach Wiedereinsatz vorhanden. Es bleibt festzuhalten, dass die Zuchtwertschätzung für Holsteins in Deutschland im Wesentlichen einen hohen Grad der Verlässlichkeit besitzt. Dies zeigt die große Mehrheit der Zuchtorganisationen, für die sehr stabile Entwicklungen der Zuchtwertschätzungen vom Testeinsatz zum Wiedereinsatz gefunden werden.

\section{Schlussfolgerungen}

Die vier untersuchten Verbandsgebiete Schleswig-Holstein, Niedersachsen-Hannover, Berlin-Brandenburg und Mecklenburg-Vorpommern können als exemplarisch für die 
deutsche Holsteinzucht angesehen werden. Der genetische Fortschritt in der unselektierten Stichprobe aller MLP-Kühe liegt auf einem vergleichsweise hohen Niveau und entspricht dem für die US-Population ausgewiesenen Zuchtfortschritt. Hinsichtlich der Ausnutzung der geprüften Bullen zeigt sich, dass manche Bullen zwar stark ausgenutzt werden, aber insgesamt eine Vielzahl von Bullen in einen allerdings meist beschränkten Wiedereinsatz geht. Dies hat zwar hinsichtlich der Erhaltung der genetischen Vielfalt Vorteile, in der starken Ausnutzung von Bullen mit Top-Zuchtwerten liegen allerdings noch Reserven, die genutzt werden sollten. Die Analyse des Vergleichs von Zuchtwerten nach Testeinsatz und Wiedereinsatz ist mit dem Problem behaftet, dass nur eine sehr kleine Stichprobe für die Auswertung zur Verfügung steht. In der Tendenz werden die Zuchtwerte aus dem Testeinsatz nach einem Wiedereinsatz der Bullen allerdings in der überwiegenden Zahl der Zuchtregionen voll bestätigt, was auf eine hohe Verlässlichkeit der deutschen Zuchtwertschätzung hinweist.

\section{Literatur}

ADLER, B.:

Aktuelle Ergebnisse aus den Rinderzuchtverbänden: Berlin-Brandenburg. In: Festkolloquium zu Ehren von Georg Schönmuth, Hrsg.: RMV und RBB, ISBN 3-922141-23-4, 1999, 69-81

AIPL:

Webseite des Animal Improvement Programs Laboratory (AIPL). http.//www.aipl.arsusda.gov; Einsicht am 23.01.2003

CDN:

Webseite des Canadian Dairy Network (CDN). http://www.cdn.ca; Einsicht am 23.01.2003

DEKKERS, J.C.M.; VANDERVOORT, G.E.; BURNSIDE, E.B.:

Optimal Size of Progeny Groups for Progeny-Testing Programs by Artificial Insemination Firms. J. Dairy Sci. 79 (1996), 2056-2070

KÖNIG, S.; SWALVE, H.H.:

Nutzung der Ergebnisse der Interbull-Zuchtwertschätzung zum Vergleich des genetischen Niveaus von Ländern. Vortragstagung der DGfZ und GfT, 20./21. September, Kiel, Paper A10. 2000

NICHOLAS, F.W.; SMITH, C.:

Increased rates of genetic change in dairy cattle by embryo transfer and splitting. Animal Production 36 (1983), 341-353.

SCHÖNMUTH, G.:

Entwicklungslinien der deutschen Schwarzbunt- und Holsteinzucht im 20. Jahrhundert. In: Festkolloquium zu Ehren von Georg Schönmuth, Hrsg.: RMV und RBB, ISBN 3-922141-23-4, 1999, 121169

SIMIANER, H.; KÖNIG, S.:

Selektionsstrategien unter Berücksichtigung der Inzucht. 3. Rinderworkshop „Neue Herausforderungen für die Rinderzucht“, 18./19. Februar, Uelzen, 2003

VAN VLECK, L.D.:

Potential genetic impact of artificial insemination, sex selection, embryo transfer, cloning and selfing in dairy cattle. In: New Technologies in Animal Breeding, Chapter 12, pp 221-241. Academic Press. 1981

Eingegangen: 29.01.2003

Akzeptiert: 20.02.2003

Anschrift der Verfasser

Prof. Dr. HERMANN H. SWALVE, KERSTIN HÖVER

Institut für Tierzucht und Tierhaltung mit Tierklinik

Martin-Luther-Universität Halle-Wittenberg

Adam-Kuckhoff-Str. 35

D-06108 Halle (Saale)

E-Mail: swalve@landw.uni-halle.de 